\title{
Formas de participación durante el prácticum en una comunidad de aula como oportunida- des de aprendizaje de la profesión de maestro
}

\section{Ways of participation during the practicum in the classroom community as opportunities to learn the teaching profession}

\author{
Bárbara Camila Toledo Fierro \\ Universitat Austral de Chile (Chile) \\ barbara.toledo01@uach.cl
}

Teresa Mauri Universitat de Barcelona, (España) teresamauri@ub.edu

Fecha de recepción 01-03-2018 Fecha de aceptación 11-04-2018 de aula como oportunidades de aprendizaje de la profesión de maestro. Revista Prácticum, 3(1), 20-33. 
Formas de participación durante el prácticum en una comunidad de aula como oportunidades de aprendizaje de la profesión de maestro.

\section{Resumen}

Este artículo presenta algunos de los resultados de una investigación centrada en conocer las formas de participación conjunta de una maestra practicante y una maestra tutora en una comunidad de aula durante el prácticum. La investigación se inscribe en una perspectiva sociocultural del aprendizaje entendido como participación. La metodología corresponde a un estudio de caso único conformado por una maestra de Educación Infantil, dieciocho alumnos y una estudiante de prácticum. Se analizan quince jornadas de aula distribuidas a lo largo de un semestre escolar. Los resultados permiten identificar nueve formas de participación conjunta diferentes, presentes durante el prácticum, que para la estudiante en prácticas representan grados variados de implicación y co-responsabilidad en dicha comunidad de aula. La distribución de las diferentes formas de participación a lo largo del prácticum no sigue, como cabría esperar, una tendencia creciente de menos a más participación por parte de la practicante, sino que muestra oscilaciones importantes que comportan avances y retrocesos en el grado de implicación en las tareas del aula, incluso en aquellas en las que ya había logrado cotas de implicación elevadas. Dichas oscilaciones se explican de acuerdo a aspectos concretos de la práctica sociocultural del aula en la cual ocurren.

\section{Abstract}

This article presents some of the results of an investigation focused on knowing the forms of joint participation of a student teacher and a tutor in a classroom community during the practicum. The research is part of a sociocultural perspective of learning understood as participation. The methodology corresponds to a singular case study consisting of a teacher of Early Childhood Education, eighteen students and a student teacher. Fifteen classroom sessions distributed throughout a semester are analysed. The results allows the identification of nine different forms of joint participation, present during the practicum, which for the student teacher represent varying degrees of involvement and co-responsibility in said classroom community. The distribution of the different forms of participation throughout the practicum does not follow, as would be expected, a growing trend, from less to more participation of the student teacher, but it shows important oscillations that involve advances and setbacks in the degree of involvement in the tasks of the classroom, even in those in which she had already achieved high levels of involvement. These oscillations are explained according to specific aspects of the sociocultural practice of the classroom in which they occur.

\section{Palabras clave}

Participación, prácticum, interacción, estudiante de maestro, aprendizaje observacional.

\section{Keywords}

Participation, practicum, interaction, student teaching, observational learning. 


\section{Introducción}

En el marco de la formación inicial del profesorado, el prácticum se considera un espacio educativo orientado principalmente a proporcionar a los estudiantes una experiencia de aprendizaje en un contexto profesional específico, que favorezca su futura incorporación al mismo. El valor formativo de este periodo proviene de las posibilidades que brinda a los estudiantes de acceder, integrarse y participar en escenarios de actividad auténtica. Para lograr que esa formación se dé realmente, cada institución universitaria propone un modelo de prácticum sustentado teóricamente y que responde a criterios organizativos y de gestión específicos. En este artículo, pretendemos conocer las oportunidades de aprendizaje de que dispone un estudiante en prácticas por su incorporación al aula escolar asignada. En consecuencia, más que en analizar un modelo formativo específico, nos focalizaremos en conocer y analizar dichas oportunidades de aprendizaje por la incorporación del estudiante en dichas prácticas.

Para ello, en primer lugar partiremos de considerar tres planos de análisis de las prácticas socioculturales características de la comunidad de aula (Rogoff, 1995): el plano comunitario, el plano interpersonal y el plano individual.

El plano comunitario se entiende (Coll, Bustos y Engel, 2008) como una configuración social donde los integrantes poseen intereses comunes y comparten convenciones y reglas implícitas o explicitas, construyendo conjuntamente una identidad y prácticas sociales a través de la interacción y la comunicación en el contexto de un espacio común. En este sentido, al considerar el aula como comunidad se pone el acento en esta como un lugar social, de actuación conjunta, que ofrece al estudiante de prácticum un potencial acceso a las prácticas que se construyen y a las competencias -en este caso, profesionales- que la participación en dichas prácticas contribuye a desarrollar (Wenger, 2001).

El plano interpersonal lo entendemos como el conjunto de posibilidades que los demás integrantes de la comunidad de aula ofrecen al estudiante de prácticum de participar e implicarse en las prácticas que la caracterizan. Al hablar de prácticas consideramos estas como ámbitos mismos de actividad conjunta donde los integrantes de la comunidad actúan e interactúan (Schatzki, 2002, 2010), es decir, espacios de actividad conjunta organizados y formalizados en actividades características que se producen en un tiempo y lugar determinado y componen el quehacer diario de los miembros de la comunidad. Dichas prácticas se concretan y definen por la actividad misma de los participantes mientras la llevan a cabo. Young (2008) define las prácticas como actividades rutinarias vinculadas a la estructura de la comunidad y al significado que esta les otorga.

Finalmente, el plano individual lo comprendemos como la continuidad y discontinuidad de las actuaciones individuales llevadas a cabo por el estudiante de prácticum en el marco de interacción con los demás, y que suponen diferentes formas de interpretar y responder en la actividad conjunta que se lleva a cabo, así como distintos grados de implicación en la misma.

En segundo lugar, estrechamente relacionado con lo anterior, partiremos de considerar el aprendizaje como participación desde una perspectiva sociocultural. Desde esta perspectiva, el estudiante de prácticum puede ser visto como alguien recién llegado a un contexto escolar de aula que le es nuevo y en el que se ve en la necesidad de implicarse en las prácticas socioculturales que lo caracterizan. En consecuencia, su aprendizaje puede considerarse como un proceso situado basado en su incorporación participativa en esas prácticas en interacción con los demás miembros de la comunidad de aula en que se inserta (Engeström, 2001; Lave y Wenger, 1991; Matusov, 1998; Rogoff, 1995). 
Formas de participación durante el prácticum en una comunidad de aula como oportunidades de aprendizaje de la profesión de maestro.

Asumimos también que el practicante aprende participando en dichas prácticas de formas diferentes y que cada forma de participación se relaciona con un cambio en la distribución del trabajo, el grado de responsabilidad en la actividad que se desarrolla y en las actuaciones individuales de los participantes (Matusov, 1998). En consecuencia, en la consideración del aprendizaje como participación nos alejamos de un enfoque teórico antropológico, que propone considerarlo como el desarrollo de una participación progresiva estructurada en términos de periferia y centro (Lave y Wenger, 1991, 2002; Wenger, 2001). Más bien, coincidimos con Edwards (2005) en considerar que un estudiante de prácticum necesariamente no participa inicialmente en la periferia de las prácticas del aula para luego, progresivamente, desplazarse hacia el centro de esas prácticas. Así, asumimos la existencia de posiciones múltiples, variadas y de formas más o menos involucradas de participar en las prácticas socioculturales características de la comunidad de aula que no siguen una dinámica estructural desde la periferia hacia el centro.

De acuerdo con lo establecido anteriormente, proponemos que las prácticas socioculturales características de la comunidad del aula son el ámbito de actividad en que se concreta la participación del estudiante de prácticum, es decir, aquellas prácticas que poseen un valor y significado dentro de la comunidad, que tienen un objetivo o finalidad determinada y que están abiertas a la observación y al análisis de los implicados. Las prácticas socioculturales disponibles pueden ser vistas como oportunidades de aprendizaje utilizables por el estudiante, y el aprendizaje puede analizarse teniendo en cuenta tanto su implicación en las mismas, como la trasformación de su participación a lo largo del prácticum. Poner el foco en la trasformación de la participación supone considerar las distintas maneras en que dicho estudiante participa en las prácticas características de la comunidad de aula, lo cual implica dar cuenta de una continua remodelación de las interacciones con los demás, así como el desarrollo de nuevas habilidades y de nuevas capacidades para implicarse en la actividad del aula. Es decir, se trata de reconocer y poner de relieve la participación de diferentes maneras en las distintas prácticas socioculturales características de la comunidad de aula del estudiante de prácticas, a través del análisis de las actuaciones articuladas con las actuaciones de otros, que toman diferentes formas interactivas a lo largo del prácticum.

\section{Método}

\subsection{Diseño}

Tanto la finalidad como los planteamientos teóricos de este trabajo ponen el foco en las distintas formas de participación construidas por los miembros en interacción en las prácticas socioculturales características de una comunidad de aula, durante un proceso de formación profesional como es el periodo de prácticum. En coherencia con este enfoque, nuestro interés se sitúa en analizar las particularidades y complejidades de un caso singular con el objetivo de comprender su actividad mediante la observación y recogida de datos en el entorno natural. Como señala Yin (2006), a través de un estudio de caso es posible iluminar una situación particular y conseguir un entendimiento profundo y de primera fuente de dicha situación. 


\subsection{Muestra}

Considerando el objetivo de este trabajo se buscó llevar a cabo el estudio en el contexto de una comunidad de aula perteneciente a un centro escolar donde un estudiante de prácticum llevara a cabo dicho periodo de formación.

Resultó pertinente seleccionar el caso de estudio considerando, principalmente, dos criterios: (i) el nivel académico, la actitud ante el aprendizaje y la disposición a participar del estudiante de prácticum; y (ii) la calidad del centro escolar de práctica como un contexto de buenas prácticas de formación inicial docente. A través de un informante experto se identificó una estudiante idónea y una escuela reconocida por llevar a cabo buenas prácticas en la formación de maestros. Siguiendo estos criterios de selección, se escogió una estudiante de cuarto curso del Grado de Educación Infantil a quien le correspondió cursar la asignatura de Prácticum II durante el primer trimestre del curso académico, en un aula de Educación Infantil del nivel educativo P5 de la cual formaban parte una maestra tutora, dieciocho alumnos entre 4 y 5 años de edad, y cuando correspondió, una maestra de apoyo o una maestra de psicomotricidad.

Antes de iniciar la recogida de datos, se obtuvo el consentimiento informado de todos los participantes del estudio, incluyendo la autorización de fotografías, vídeos y cesión de imagen de menores firmada por los padres de los alumnos involucrados.

\subsection{Procedimiento de recogida de datos}

Para el caso seleccionado, las unidades de observación y registro supusieron una estancia intensiva y una continuidad temporal lo suficientemente amplia, de modo que permitiese observar y registrar la interacción de los participantes dentro de la comunidad de aula, su evolución momento a momento y a lo largo del prácticum. Así, se registró un conjunto de jornadas de aula continuadas representativas del periodo de prácticum, que resultaran en un volumen de datos abarcable en el marco del estudio.

Se registraron 15 jornadas de aula (en adelante JdA), 5 pertenecientes a la fase inicial, 5 a la fase de desarrollo, y 5 a la fase final del prácticum. Las $15 \mathrm{JdA}$ estaban dirigidas a la enseñanza y aprendizaje de diversas competencias y contenidos curriculares del nivel educativo correspondiente. Las JdA fueron registradas mediante audio, vídeo y notas de campo, correspondiendo al total de horas que los alumnos permanecieron dentro del aula durante una mañana habitual de clases, formando un cuerpo de datos de, aproximadamente, 45 horas de registro de la actividad de aula. 
Formas de participación durante el prácticum en una comunidad de aula como oportunidades de aprendizaje de la profesión de maestro.

\subsection{Procedimiento de análisis de datos}

El análisis de los datos recogidos fue llevado a cabo siguiendo un modelo construido para este estudio, y que se concreta en dos niveles de análisis con finalidades específicas pero vinculados entre sí. Para efectos del presente trabajo, se detalla únicamente el primer nivel de análisis que ha permitido la consecución del objetivo aquí presentado. Este primer nivel fue elaborado con la finalidad de identificar y caracterizar, en primer término, las diferentes prácticas socioculturales características de la comunidad de aula, y en segundo término, situadas en estas prácticas, de identificar y caracterizar las distintas formas de participación que la maestra tutora y la estudiante de prácticum configuran y la estructura y evolución de dichas formas a lo largo del prácticum.

Así, un primer foco de caracterización de los datos se basó en el modelo de análisis de la interactividad desarrollado por Coll y colaboradores (Colomina, Onrubia y Rochera, 2001; Coll, Onrubia y Mauri, 2008).

Sobre los resultados de este primer foco, se llevó a cabo un segundo foco de caracterización, construido principalmente, con base en el trabajo de Fillietaz, Trebert y Rémery (2012). Este segundo foco se centró en la manera en que cada maestra -considerando a la estudiante como maestra practicante- se implica en el desarrollo de las distintas prácticas socioculturales, es decir, centrándonos por separado, en las actuaciones que la maestra tutora y la maestra practicante ejecutan al interactuar entre sí y con los alumnos en el contexto de una práctica característica de la comunidad de aula.

Gracias al primer foco de caracterización, contábamos con una descripción detallada de las actuaciones típicas que caracterizan cada práctica sociocultural de la comunidad de aula. Con el segundo foco identificamos momento a momento si dichas actuaciones eran ejecutadas por cada maestra de manera: (a) global: orientada al grupo clase o a varios alumnos a la vez y en voz alta; (b) focalizada: orientada a un alumno y en voz baja; (c) sistemática: ejecutada repetidamente a lo largo de la actividad; (d) esporádica: ejecutada ocasionalmente a lo largo de la actividad; y/o (e) puntual: realizada una sola vez a lo largo de la actividad. A partir de lo anterior, se configuró el grado y tipo de implicación que cada maestra desempeñaba en el marco de una práctica sociocultural determinada.

\section{Resultados}

De acuerdo al objetivo que vertebra este artículo, a continuación se presentan los principales resultados relacionados con las distintas formas de participación construidas por las maestras en el marco de las prácticas socioculturales características de la comunidad de aula estudiada.

En primer lugar se presenta una breve descripción y caracterización de cada una de las formas de participación identificadas. En segundo lugar, se presentan un resumen de la estructura y el proceso de evolución que siguen estas formas de participación en el trascurso de las jornadas de aula y de manera global durante el prácticum. 


\subsection{Formas de participación construidas por las maestras}

Se identificaron nueve formas de participación distintas (en adelante FP) que permiten describir y conocer diferentes tipos de interacción entre las maestras y grados de implicación en las prácticas socioculturales de la comunidad de aula.

- Presencia sin intervención o ausencia (NI). La dinámica de participación se caracteriza porque la maestra tutora asume el liderazgo, control y responsabilidad total de la gestión de la participación, contenido y elaboración de significados en torno a la tarea que se desarrolla con los alumnos. Entretanto, la maestra practicante se encuentra presente dentro del aula pero no se implica de ninguna forma en la actividad que se está desarrollando con el gran grupo, o se encuentra fuera del aula (por un período de tiempo significativo en términos interpretativos).

- Observación sin/con intervención puntual (OIP). La dinámica de participación se caracteriza porque la maestra tutora lidera, controla y tiene la responsabilidad primordial sobre la gestión de la participación, contenidos y elaboración de significados en relación a la tarea que se ejecuta con los alumnos, mientras la maestra practicante adopta una postura de observadora externa, sin intervenir global, sistemática ni esporádicamente en el desarrollo de la actividad. Es la maestra tutora el eje y destinataria directa de las interacciones con los alumnos y la maestra practicante ocupa un papel de «testigo» de dichas interacciones, pudiendo llevar a cabo de manera puntual y focalizada, pequeñas intervenciones, regresando rápidamente a su lugar de observadora.

- Co-participación con apoyo (CpA). La dinámica de participación se caracteriza porque es primordialmente la maestra tutora quien posee el liderazgo, el mando y la responsabilidad tanto de la gestión de la participación, como de la elaboración de los significados relacionados con la tarea que se desarrolla con los alumnos. No obstante, la maestra practicante, junto con cumplir un papel de observadora de la actividad, toma parte de esta mediante la ejecución esporádica de una o más actuaciones típicas de la práctica sociocultural en cuestión, pero sin involucrarse global ni sistemática en ella. Se mantienen los roles asimétricos entre ambas maestras.

- Co-participación con implicación (CpI). La dinámica de participación se caracteriza porque se equilibra la asimetría de roles entre ambas maestras, y se reconoce un mayor grado de abertura en la actividad que se desarrolla con los alumnos, ya sea por parte de la maestra tutora, o por iniciativa de la maestra practicante. La maestra tutora posee el liderazgo, dominio y responsabilidad de la actividad, mientras que la maestra practicante se implica global y sistemáticamente en ella, ejecutando activamente las actuaciones típicas que caracterizan la práctica en cuestión, relacionadas principalmente con la supervisión del trabajo desarrollado por los alumnos, la ayuda individual en el desarrollo de este, el recordatorio de las instrucciones de la tarea, y la manipulación de los recursos materiales. Ambas maestras se distribuyen roles complementarios: la maestra tutora mantiene la dirección y dominio sobre la actividad, y la maestra practicante ejecuta actuaciones similares y/o complementarias en coordinación con la maestra tutora. 
Formas de participación durante el prácticum en una comunidad de aula como oportunidades de aprendizaje de la profesión de maestro.

- Co-participación con delegación (CpD). La dinámica de participación se caracteriza porque la maestra tutora posee el liderazgo, el mando y la responsabilidad sobre la gestión de la participación, contenidos y elaboración de significados en torno a una tarea que se desarrolla con el gran grupo. En tanto, simultáneamente la maestra practicante lidera y es responsable de una tarea paralela que se desarrolla con uno o más alumnos. La maestra tutora es la interlocutora principal y destinataria directa de las interacciones con la mayoría del grupo clase, mientras la maestra practicante ocupa el rol de interlocutora y destinataria directa de las interacciones con los alumnos que están ejecutando la tarea que ella dirige.

- Co-participación con distribución (CpDt). La dinámica de participación se caracteriza por una simetría observada en las actuaciones de ambas maestras, distribuyéndose el liderazgo, control y responsabilidad sobre la gestión de la participación, contenidos y elaboración de significados de una actividad paralela, cada una con un pequeño grupo de alumnos. Cada maestra dirige y domina la gestión de la tarea que le corresponde, ocupando frente a un pequeño grupo de alumnos, el papel de interlocutora principal y destinataria directa de las interacciones.

- Co-participación con traspaso con implicación (CpTI). La dinámica de participación se caracteriza porque es la maestra practicante quien lidera, toma el mando y la responsabilidad de la gestión de la participación, contenidos y elaboración de significados en relación a la tarea. Entretanto, la maestra tutora ocupa un rol secundario, ejecutando actuaciones globales y focalizadas, alineadas con las actuaciones de la maestra practicante y complementándolas. De esta manera, la maestra practicante ocupa un espacio de autonomía, dirigiendo y guiando sistemática y globalmente la actividad que desarrolla con los alumnos, cumpliendo el rol de interlocutora principal y destinataria directa de las interacciones con estos. Por su parte, la maestra tutora, junto con observar el desarrollo de la actividad, interviene principalmente, para regular la participación de los alumnos, profundizar los contenidos y controlar el tiempo.

- Co-participación con traspaso con apoyo (CpTA). La dinámica de participación se caracteriza porque, al igual que CpTI, la maestra practicante posee el liderazgo y toma el mando y responsabilidad de la gestión de la participación, contenidos y elaboración de significados de la actividad que desarrolla con los alumnos. Mientras, la maestra tutora adopta una postura de observadora externa, sin intervenir sistemática ni globalmente en el desarrollo de esta. La maestra practicante ocupa un espacio de autonomía, dirigiendo y guiando sistemática y globalmente la actividad desarrollada con los alumnos, cumpliendo un rol de interlocutora principal y destinataria directa de las interacciones con estos. La maestra tutora intercala periodos de observación, ejecución de actuaciones esporádicas y focalizadas relacionadas principalmente con la gestión de la participación de los alumnos, y ejecución de actuaciones que no pertenecen a la actividad desarrollada con los alumnos.

- Co-participación con traspaso sin intervención (CpTsI). La dinámica de participación se caracteriza porque la maestra practicante posee el total liderazgo y toma de mando y responsabilidad de la gestión de la participación, contenidos y elaboración de significados en la actividad que se desarrolla con los alumnos. La maestra tutora no interviene en esta -salvo observaciones o intervenciones puntuales-, ejecutando actuaciones que no pertenecen a dicha actividad, o se encuentra fuera del aula (por un período de tiempo significativo en términos interpretativos). 
Cada una de estas FP corresponde a un grado de implicación distinto de la estudiante de prácticum en una práctica sociocultural determinada, siempre en interacción con las actuaciones de la maestra tutora y los alumnos. Como muestra la Ilustración 1, estas nueve FP forman una escala ascendente, donde el primer peldaño corresponde al mínimo grado de implicación de la maestra practicante -Presencia sin intervención o ausencia- y el último representa el máximo grado de implicación -Co-participación con traspaso sin intervención- existiendo entre uno y otro siete formas de participación distintas.

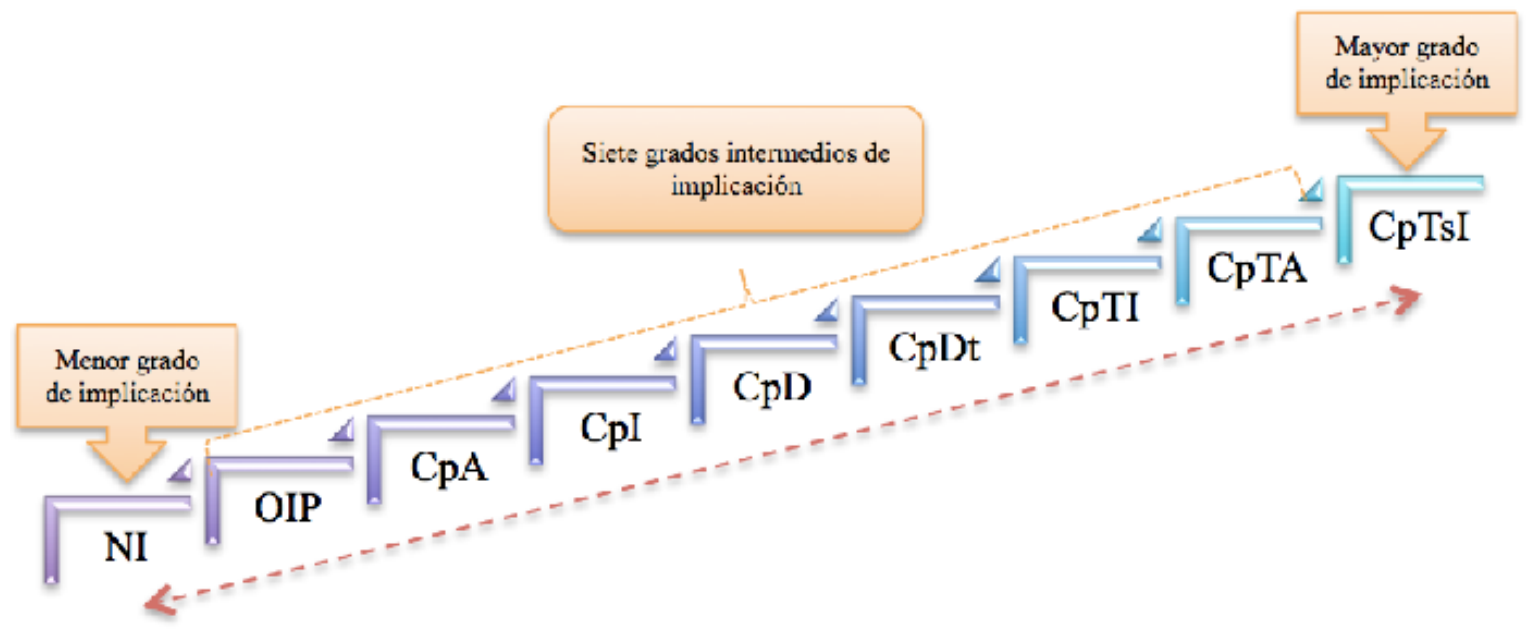

Ilustración 1: Formas de participación construidas por la maestra tutora y la maestra practicante en el marco de distintas prácticas socioculturales de la comunidad de aula, Elaboración propia.

\subsection{Evolución a lo largo del prácticum de las formas de participa- ción construidas por las maestras}

A continuación se sintetizan las principales ideas respecto de la evolución de las distintas FP construidas por la maestra tutora y la maestra practicante en la comunidad de aula analizada, lo cual también se resume en la Tabla 1.

De las nueve FP identificadas, en promedio ocurren siete FP distintas en cada JdA analizada. En conjunto, estas FP se distribuyen a lo largo de cada jornada con una frecuencia de aparición aproximada de 19 veces.

Parece interesante destacar las FP que resultan más significativas en términos de frecuencia de aparición y tiempo de duración, tanto intra como inter jornadas a lo largo del prácticum. La FP OIP corresponde a la forma de participación que, con diferencia, ocurre en mayor número de veces a lo largo de las tres fases del periodo de prácticum y ocupa el mayor porcentaje de tiempo global. Esta FP se observa en las quince JdA y ocurre un total de noventa veces a lo largo del prácticum, representando casi un 35\% del tiempo total de este. En once JdA esta FP supone entre un $30 \%$ hasta casi un $60 \%$ del tiempo de la jornada en la cual se presenta.

Otra FP que resulta significativa corresponde a la FP CpI que ocurre al menos una vez en las quince jornadas de aula analizadas. Esta FP se da un total de cincuenta y una veces a lo largo del prácticum y ocupa más del $20 \%$ del tiempo total de este. En seis jornadas esta FP ocupa más del 30\% del tiempo total. 
Formas de participación durante el prácticum en una comunidad de aula como oportunidades de aprendizaje de la profesión de maestro.

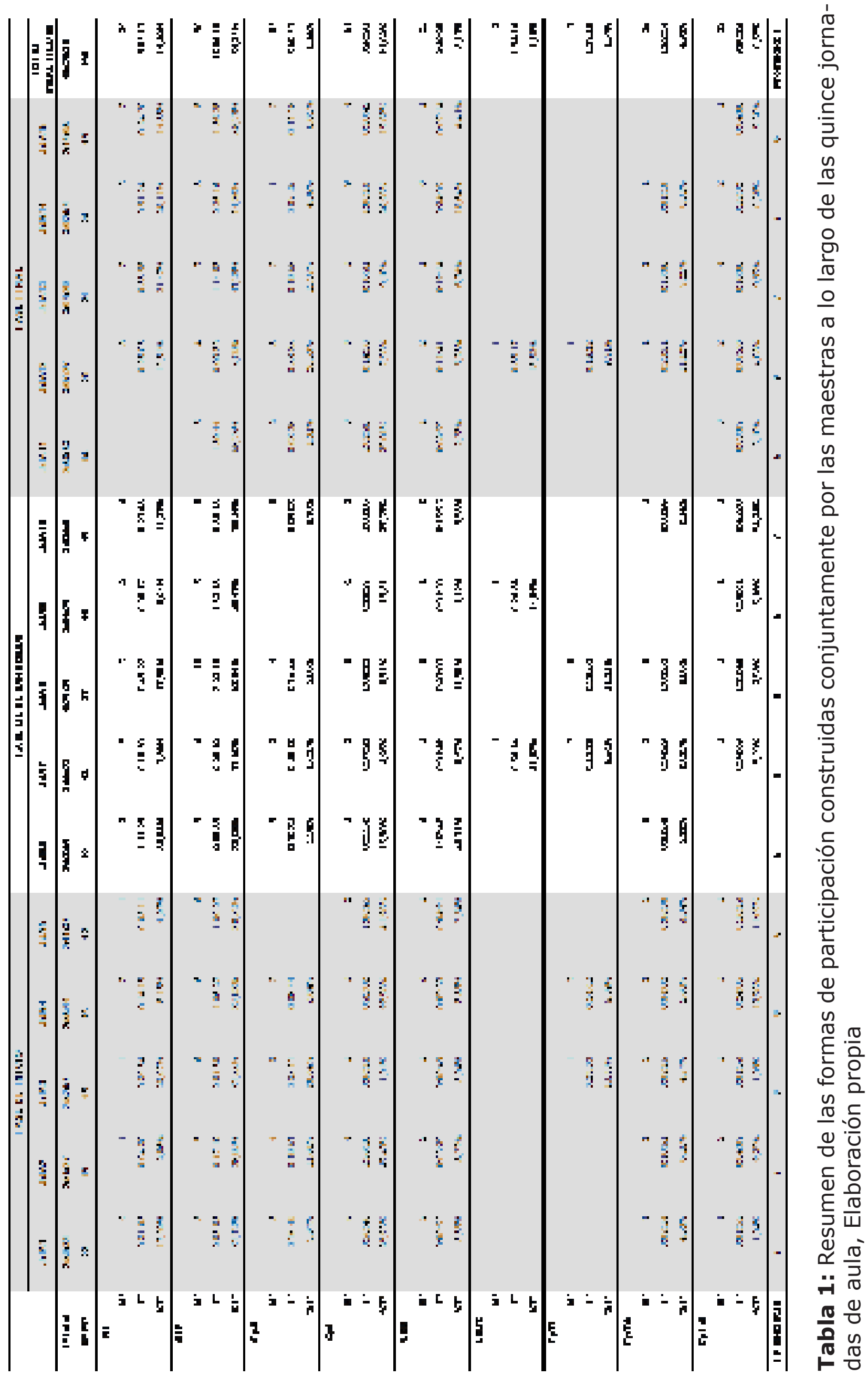


Llama también la atención que la FP NI, ocupa el tercer lugar tanto en relación a la frecuencia de aparición como en la cantidad de tiempo que representa a nivel del prácticum. La frecuencia de aparición de esta FP es bastante alta, ocurre treinta y nueve veces a lo largo del prácticum, y el tiempo de duración corresponde a un $15 \%$. Sin embargo, lo más interesante es el porcentaje de tiempo que representa esta FP al interior de las jornadas de aula, pues, si bien el tiempo de duración no resulta estable ni tampoco varía siguiendo alguna tendencia reconocible, podemos observar que el porcentaje de tiempo aumenta en la fase de desarrollo y en la fase final respecto de la fase de inicio.

En cuarto lugar, podemos ver que la FP CPA es una forma de participación que, representa un porcentaje de tiempo total apreciablemente más bajo $(8 \%)$, pero su frecuencia de aparición es medianamente alta: treinta y dos veces en referencia al prácticum. El tiempo de duración de esta FP varía notablemente de una jornada a otra y no sigue ninguna tendencia observable.

Respecto a las tres FP que suponen los grados más alto de implicación de la maestra practicante -CPTI, CPTA, CpTSI-, el tiempo de duración de cada una varía medianamente. CpTI ocupa un 3,57\% del tiempo total, CpTA corresponde a un 4,39\%, y CPTSI representa un 6,62\% del tiempo total. Estas tres FP se encuentran entre las menos configuradas por las maestras de esta comunidad de aula. Sin embargo, es posible destacar que cuando se da la FP CPTI corresponde a un porcentaje de tiempo significativo respecto del tiempo total de la JdA. Por su parte, la FP CPTA aparece en catorce ocasiones, distribuidas en once JdA, sin embargo, pese a que esta forma de participación ocurre con mayor regularidad y frecuencia, cuando aparece lo hace durante cortos periodos de tiempo.

Algo similar ocurre con la FP CPTSI. Salvo en la JdA6, en las otras catorce jornadas esta FP ocurre al menos una vez, por lo que ocupa el quinto puesto en frecuencia de ocurrencia total a lo largo del prácticum (veintiocho veces). No obstante, en ocho de las catorce JdA en las que ocurre, el tiempo de duración corresponde a un 5\% o menos.

Ahora bien, si observamos de manera conjunta estas últimas tres FP, resulta interesante constatar que desde el inicio hasta el final del prácticum, ocurre al menos una de ellas, que en conjunto representan un $15 \%$ del tiempo total del prácticum.

\section{Discusión y conclusiones}

Cada una de las nueve FP se corresponde con un grado distinto de implicación de la maestra practicante en la práctica sociocultural que se desarrolla, las cuales transitan desde una mínima o nula implicación en la actividad, hasta una implicación total y ejecución completa de una práctica sociocultural específica.

Sin embargo, y siguiendo a autores como Edwards (2005), Hodkinson y Hodkinson (2004) y Mauri, Clarà, Ginesta y Colomina (2013) las diferentes FP, su frecuencia de aparición y su distribución tanto durante el prácticum, no pueden ser comprendidas desde una lógica de «Participación Periférica Legítima» (Lave y Wenger, 1991, 2002; Wenger, 2001). En su rol de maestra practicante, la estudiante participa de forma «periférica» y/o «central» independientemente de la fase temporal del prácticum. Es más, las nueve FP aparecen distribuidas heterogéneamente tanto intra como inter jornadas, observándose incluso las nueve formas distintas en una misma jornada de aula (JdA7 y JdA12). 
Formas de participación durante el prácticum en una comunidad de aula como oportunidades de aprendizaje de la profesión de maestro.

En consecuencia, las FP y su evolución parecen obedecer a otros factores. Así por ejemplo, la FP OIP es la forma más significativa de participar de la estudiante de prácticum en las prácticas socioculturales de la comunidad de aula analizada. Esta notoria superioridad otorga relevancia a un aspecto que ha recibido poca atención en la investigación educativa, la observación como una valiosa fuente de aprendizaje (Rogoff, Paradise, Mejía, Correa-Chávez y Angelillo, 2003). Una observación como una forma de participación, llevada a cabo como anticipación a la implicación en la actividad que se observa.

Estos resultados apoyan lo planteado por Billett (2014) acerca del aprendizaje mimético en situaciones de práctica: observación-imitación-ensayo. Este autor plantea que es a través de los sucesivos intentos de reproducir actividades observadas que se construye, al mismo tiempo, el aprendizaje de dichas actividades. Lo anterior es aún más potente cuando este proceso se lleva a cabo en circunstancias donde existen posibilidades de que el aprendiz observe intensivamente los desempeños requeridos, como es el caso de lo que ocurre en la comunidad de aula analizada.

El proceso de observación-imitación-ensayo nos permite explicar no solo la significatividad de la FP OIP, sino también lo que ocurre, por ejemplo, con la FP CpI, la cual supone una distribución y/o duplicación por parte de las maestras de las actuaciones de las prácticas socioculturales en las cuales ocurre, manteniendo la maestra tutora el dominio de la actividad con los alumnos, resultando ser el escenario idóneo en el cual la maestra practicante ensaya o repite las actuaciones previamente observadas, refinando y perfeccionando así su desempeño (Billett, 2014).

Algo parecido ocurre con el resto de FP (exceptuando NI) coincidiendo que la manera en que la maestra practicante ejecuta las actuaciones propias de la práctica sociocultural, corresponde siempre a maneras idénticas o muy similares a cómo las ejecuta la maestra tutora, diferenciándose solo en la forma (global o focalizada) y la periodicidad (sistemática, esporádica o puntual).

A modo de conclusión, las distintas FP construidas por la maestras se configuran en función de cómo cada una ejecuta, contribuye y hace suyo el patrón de actuaciones que caracteriza cada práctica sociocultural, con especial interés en la estudiante de prácticum. La frecuencia de aparición, distribución y evolución de estas FP es variable y heterogénea. Visto desde el punto de vista de la maestra practicante, la trayectoria que siguen las distintas FP no obedece a una línea de menor a mayor implicación a lo largo del periodo de prácticum. La maestra practicante participa en un nivel mínimo y máximo -y los intermedios- en la fases de inicio, desarrollo y final del prácticum.

Así, las principales oportunidades de aprendizaje disponibles para la practicante durante su participación en las prácticas socioculturales de la comunidad de aula se basan principalmente, en observar y escuchar las actividades típicas que la tutora desarrolla con los alumnos y llevar a cabo estas actividades en colaboración con la maestra tutora.

Finalmente, contamos con una primera aproximación a las formas de participación construidas por las maestras. No obstante, si bien los resultados presentados aquí nos permiten conocer el abanico de posibilidades de participación para la estudiante de prácticum en una comunidad de aula, como hemos señalado en trabajos anteriores (Toledo y Mauri, 2014, 2015, 2016), la evolución de las FP debe interpretarse ensamblada a las prácticas socioculturales características de dicha comunidad. Es necesario situar las FP en el contexto concreto de las actividades características del aula en la cuales ocurren, ya que estas últimas circunscriben el grado de implicación permitido y las oportunidades de acción disponibles para la practicante. 


\section{Bibliografia}

Billett, S. (2014). Mimetic learning at work. Learning in the circumstances of practice. Dordrecht, Países Bajos: Springer. doi: 10.1007/978-3-319-09277-5 Coll, C., Bustos, A., y Engel, A. (2008). Las comunidades virtuales de aprendiza je. En C. Coll, y C. Monereo (Eds.), Psicología de la educación virtual (pp. 299- 320). Madrid, España: Morata.

Coll, C., Onrubia, J., \& Mauri, T. (2008). Ayudar a aprender en contextos educativos: el ejercicio de la influencia educativa y el análisis de la enseñanza. Revista de Educación, Mayo-Agosto, 33-70. Recuperado de http://www.revistaeducacion.mec.es/re346/re346_02.pdf

Colomina, R., Onrubia, J. y Rochera, M. (2001). Interactividad, mecanismos de influencia educativa y construcción del conocimiento en el aula. En C. Coll, J. Palacios, y M. Marchesi (Eds.), Desarrollo psicológico y educación. Volumen II. Psicología de la educación escolar (pp. 437-458). Madrid, España: Alianza.

Edwards, A. (2005). Let's get beyond community and practice: The many meanings of learning by participating. Curriculum Journal, 16, 49-65. doi: https://doi.org/10.1080/0958517042000336809

Engeström, Y. (2001). Expansive learning at work: Toward an activity theoretical reconceptualization. Journal of education and work, 14, 133-156. Recuperado de http://www.tandfonline.com/doi/pdf/10.1080/13639080020028747

Filliettaz, L., Trébert, D., \& Rémery, V. (2012). Relation tutorale et configuration de participation à l'interaction: le cas de la formation professionnelle des éducatrices et éducateurs de l'enfance [Relación tutorial y configuración de la participación en la interacción: el caso de la formación profesional de educadores y educadoras de infantes]. Ponencia presentada en el $2^{\circ}$ Coloquio internacional de didáctica profesional, aprendizaje y desarrollo profesional, Universidad de Nantes, Nantes, Francia. Recuperado de https://archive-ouverte.unige.ch/unige:27171

Hodkinson, P., y Hodkinson, H. (2004). A constructive critique of communities of practice: moving beyond Lave and Wenger. Paper presentado en Integrating work and learning - contemporary issues' seminar series 2004. Sidney, Australia. Recuperado de http://www.voced.edu.au/content/ngv\%3A37993

Lave, J., y Wenger, E. (1991). Situated learning: Legitimate peripheral participation. Cambridge, Reino Unido: Cambridge University Press.

Lave, J., y Wenger, E. (2002). Legitimate peripheral participation in communities of practice. En M. Lea, y K. Nicoll (Eds.), Distributed Learning. Social and cultural approaches to practice (pp. 56-63). London, Inglaterra: Routledge.

Matusov, E. (1998). When solo activity is not privileged: Participation and internalization models of development. Human Development, 41, 326-349. doi: http://dx.doi.org/10.1159/000022595 
Formas de participación durante el prácticum en una comunidad de aula como oportunidades de aprendizaje de la profesión de maestro.

Mauri,T., Clarà, M., Ginesta, A. y Colomina, R. (2013). La contribución al aprendizaje en el lugar de trabajo de los equipos docentes universitarios. Un estudio exploratorio. Infancia y Aprendizaje, 36(3), 341-360. doi: https://doi.org/10.1174/021037013807533025

Rogoff, B. (1995). Observing sociocultural activity on three planes: Participatory appropriation, guided participation and apprenticeship. En J. Wertsch, P. del Río, y A. Álvarez (Eds.), Sociocultural studies of mind (pp. 139-164). Cambridge, Reino Unido: Cambridge University Press.

Rogoff, B., Paradise, R., Arauz, R. M., Correa-Chavez, M., \& Angelillo, C. (2003). Firsthand learning through intent participation. Annual Review of Psychology, 54, 175-203. doi: https://doi.org/10.1146/annurev.psych.54.101601.145118

Schatzki, T.R. (2002). The site of the social: A philosophical account of the constitution of social life and change. Philadelphia, Estados Unidos: University of Pennsylvania Press.

Schatzki, T.R. (2010). Materiality and social life. Nature and Culture, 5, 123-149. doi: http://dx.doi.org/10.3167/nc.2010.050202

Toledo, B., y Mauri, T. (2014). Construcción social de las prácticas de una comunidad de aula de educación infantil: Análisis de la actividad conjunta de la maestra tutora, maestra practicante y alumnos/as. En M. A. Flores, C. Coutinho, y J. A. Lencastre (Comps.), Atas do congresso Formação e trabalho docente na sociedade da aprendizagem. Organizado no âmbito da International Study Association on Teachers and Teaching (ISATT) e do Centro de Investigação em Estudos da Criança (CIEC) (pp. 489-500). Braga, Portugal: CIEC, UMinho.

Toledo, B., y Mauri, T. (2015). Dinámicas de participación en una comunidad de aula: Oportunidades de aprendizaje del futuro maestro durante el período de prácticum. Papeles de Trabajo sobre Cultura, Educación y Desarrollo Humano, 11, 121-127. Recuperado de http://www.uam.es/otros/ptcedh/2015v11_pdf/v11n2sp.pdf

Toledo, B. y Mauri, T. (2016). Aprendizaje como participación durante el prácticum en un aula de Educación Infantil. Estudios Pedagógicos, 42, 343-363. doi: http://dx.doi. org/10.4067/S0718-07052016000200020

Wenger, E. (1998/2001). Comunidades de práctica. Aprendizaje, significado e identidad (G. Sánchez, Trad.; Título original: Communities of practice: Learning, meaning and identity). Barcelona: Paidós.

Yin, R. (2006). Case study methods. En J. Green, G. Camilli, y P. Elmore (Eds.), Handbook of complementary methods in education research (pp. 111-122). Mahwah, Estados Unidos: Lawrence Erlbaum y AERA.

Young, R. (2008). Chapter two: Foundations for the study of practice. Lenguage Learning, 58(2), 9-47. doi: http://dx.doi.org/10.1111/j.1467-9922.2009.00489.x 